\title{
Neural Mechanisms of Surround Attenuation and Distractor Competition in Visual Search
}

\author{
Carsten N. Boehler, ${ }^{1,2}$ John K. Tsotsos, ${ }^{3}$ Mircea A. Schoenfeld, ${ }^{1}$ Hans-Jochen Heinze, ${ }^{1}$ and Jens-Max Hopf ${ }^{1}$ \\ ${ }^{1}$ Department of Neurology, Otto-von-Guericke University and Leibniz Institute for Neurobiology, Magdeburg 39120, Germany, ${ }^{2}$ Center for Cognitive \\ Neuroscience, Duke University, Durham, North Carolina 27708, ${ }^{3}$ Centre for Vision Research and Department of Computer Science and Engineering, York \\ University, Toronto, Ontario M3J 1P3, Canada
}

Visual attention biases relevant processing in the visual system by amplifying relevant or attenuating irrelevant sensory input. A potential signature of the latter operation, referred to as surround attenuation, has recently been identified in the electromagnetic brain response of human observers performing visual search. It was found that a zone of attenuated cortical excitability surrounds the target when the search required increased spatial resolution for item discrimination. Here we address the obvious hypothesis that surround attenuation serves distractor suppression in the vicinity of the target where interference from irrelevant search items is maximal. To test this hypothesis, surround attenuation was assessed under conditions when the target was presented in isolation versus when it was surrounded by distractors. Surprisingly, substantial and indistinguishable surround attenuation was seen under both conditions, indicating that it reflects an attentional operation independent of the presence of distractors. Adding distractors in the target's surround, however, increased the amplitude of the $\mathrm{N} 2 \mathrm{pc}$ - an evoked response known to index distractor competition in visual search. Moreover, adding distractors led to a topographical change of source activity underlying the N2pc toward earlier extrastriate areas. In contrast, the topography of reduced source activity due to surround attenuation remained unaltered with and without distractors in the target's surround. We conclude that surround attenuation is not a direct consequence of the attenuation of distractors in visual search and that it dissociates from attentional operations reflected by the $\mathrm{N} 2 \mathrm{pc}$. A theoretical framework is proposed that links both operations in a common model of top-down attentional selection in visual cortex.

\section{Introduction}

There is abundant evidence indicating that visual spatial attention operates by enhancing (Hillyard et al., 1998; Maunsell and Cook, 2002) and attenuating (Rees et al., 1997; Smith et al., 2000; Vanduffel et al., 2000; Pinsk et al., 2004; Schwartz et al., 2005) neural responses in retinotopic visual cortex. Electrophysiological and fMRI research indicate that both often combine to form a center-surround profile where enhancement at the target's location is surrounded by a zone of attenuation (Slotnick et al., 2002; Müller and Kleinschmidt, 2004; Hopf et al., 2006a; Boehler et al., 2009). Hopf et al. (2006a) used MEG to analyze the passive excitability of visual cortex as a function of distance to the focus of attention in visual search. We observed that the focus of attention is flanked by a zone of attenuated excitability forming a profile that resembles a Mexican hat. A more detailed assessment of the underlying neural operations revealed that surround attenuation arises as a consequence of recurrent processing in visual cortex if the task requires spatial scrutiny for target discrimination (Boehler et al., 2009; Hopf et al., 2010). Based on those observations, one

Received Dec. 9, 2010; accepted Dec. 30, 2010.

This research was made possible by Deutsche Forschungsgemeinschaft Grant SFB779/TPA1. We thank Steven J. Luck for helpful comments and suggestions.

Correspondence should be addressed to Jens-Max Hopf, Department of Neurology, Otto-von-Guericke University, Leipziger Strasse 44, D-39120 Magdeburg, Germany. E-mail: jens-max.hopf@med.ovgu.de.

DOI:10.1523/JNEUROSCI.6406-10.2011

Copyright $\odot 2011$ the authors $\quad 0270-6474 / 11 / 315213-12 \$ 15.00 / 0$ obvious interpretation of such surround attenuation is that it provides a very effective way of improving the signal-to-noise ratio of the search process, because it suppresses the most impeding interference from nearby distractors. While this notion is intuitive and parsimonious, it has not been tested experimentally whether surround attenuation is in fact a direct consequence of competition from nearby distractors in visual search. Here we address this issue with neuromagnetic brain recordings by analyzing surround attenuation as a function of whether the search target is densely surrounded by distractors versus when it is presented without nearby distractors. Surround attenuation is assessed by measuring the size of the MEG response to a task-irrelevant probe stimulus flashed at a constant position in a search array while the target's distance to this probe varies from trial to trial. To validate the presence of attention effects due to distractors, we also assess the $\mathrm{N} 2 \mathrm{pc}-\mathrm{a}$ component of the neuroelectric/neuromagnetic brain response that is highly sensitive to competition between the target and distractors (Luck and Hillyard, 1994; Eimer, 1996; Luck et al., 1997; Woodman and Luck, 1999; Hopf et al., 2000, 2002; Hickey et al., 2009). The N2pc amplitude typically increases when the number of distractors increases (Luck et al., 1997), and source activity underlying the N2pc in ventral extrastriate cortex becomes stronger with increasing feature overlap between target and distractors (Hopf et al., 2002; Leblanc et al., 2008). Luck et al. (1997) established that the N2pc represents a population-level analog of firing suppression for 
distractor filtering in ventral extrastriate cortex of macaques performing a visual search task (Chelazzi et al., 1993, 1998).

We find-in confirming previous observations - that the N2pc amplitude increased when adding distractors in the vicinity of the search target. Surround attenuation, in contrast, remained uninfluenced, indicating that the neural operations indexed by the N2pc and surround attenuation represent different attentional operations.

\section{Materials and Methods}

Experiment 1

Subjects. The experiment was undertaken with the understanding and written consent of the subjects and approved by the ethics committee of the Otto-von-Guericke University of Magdeburg. Fifteen neurologically normal subjects took part in the experiment $(13 \mathrm{fe}$ males, mean age: 25 ) and were paid for participation. All subjects were right handed, with normal color vision and normal or correctedto-normal visual acuity.

Stimuli and probe procedure. As illustrated in Figure 1, $A$ and $B$, two different types of search frames were used that differed as to the number of distractors presented. Both contained one red and one green $\mathrm{C}$ at mirror image positions in the left and right visual field (VF). In one type of trials, referred to as "multiple-distractor trials" ( $50 \%$ of the trials), the red and green items were presented together with eight blue $\mathrm{Cs}$, four in the left and four in the right VF (Fig. $1 A)$. In the other type of trials, the red and green $C$ was presented without the blue distractors ("single-distractor trials," $50 \%$ of the trials) (Fig. $1 B$ ). For both trial types, possible item locations were aligned at an isoeccentric distance from fixation ( $8^{\circ}$ of visual angle) with five positions in each VF clustered around the two center positions halfway between the horizontal and vertical meridian. Each $\mathrm{C}$ subtended $0.8^{\circ}$ of visual angle, and spacing between Cs was constant $\left(1.35^{\circ} ; 8.2^{\circ}\right.$ radial angle). While the gap of the blue distractor Cs varied randomly in one of four directions (left, right, up, down), the gap of the red and green $\mathrm{C}$ varied between left and right, albeit independent of each other. Trial types were randomly mixed within experimental blocks. At the beginning of each trial block, either red or green was designated as the target color, and subjects were asked to report the gap orientation of the target by pressing one of two buttons with the right hand (index finger = gap left, middle finger $=$ gap right). Target color was alternated between trial blocks, but collapsed for data analysis such that only target position was retained as a condition. On each trial, the target appeared randomly at one of the five possible item positions in the left or the right VF. Each search frame was presented for $700 \mathrm{~ms}$, followed by a variable intertrial interval jittered between 650 and $850 \mathrm{~ms}$ (boxcar distribution). The spatial profile of the focus of attention was assessed by flashing $(50 \mathrm{~ms})$ a passive probe stimulus (a white ring) at a fixed (center) position of the target VF $250 \mathrm{~ms}$ after search frame onset in 50\% of the trials [frame + probe (FP) trials] (Fig. 1C,D). On the remaining $50 \%$ of the trials, no probe was presented [frame-only (FO) trials]. Because the probe always appeared at a fixed center location, its distance to the target changed from trial to trial randomly and could therefore serve as a measure of cortical excitability as a function of distance to the focus of attention. As illustrated in Figure 1,C and D, there were three target-to-probe

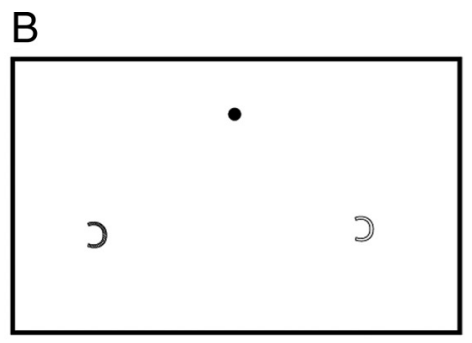

$\mathrm{D}$

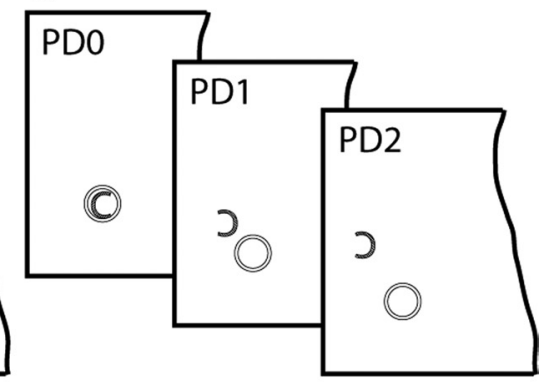

$\mathrm{F}$

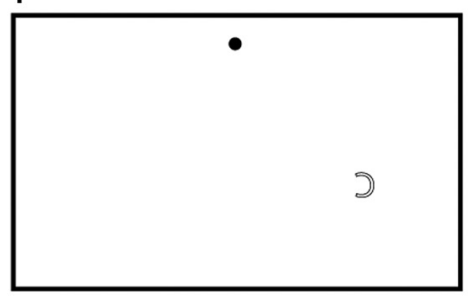

J blue Dright blue

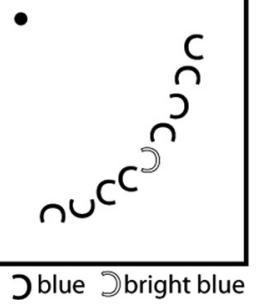

ents 1 and 2). $\boldsymbol{A}$, Example search array of the multiple-distractor condition of experiment 1 . The popout target (the red or the green $C$ ) was presented together with four blue distractors in its surround. $\boldsymbol{B}$, Example array of the single-distractor condition of experiment 1 . The target was presented together with only one in the opposite VF. C, D, Illustration of the three attention-to-probe distances in experiment 1 . When presented, the probe (the white ring) always appeared at the center item location $(\boldsymbol{C})$ or the corresponding blank location (D) in the target VF. $\boldsymbol{E}$, $\boldsymbol{F}$, Example search arrays of the multiple- $(\boldsymbol{E})$ and no- $(\boldsymbol{F})$ distractor conditions in experiment 2. Trials of the multiple- and no-distractor conditions were run in separate and alternating experimental blocks.

distances [in short, probe distances (PDs)] within each visual field, ranging from PD0 (target at the probe's location) through PD2 (target two items away from the probe). Previous studies revealed that with this stimulus setup, two positions flanking the probe's location are sufficient to reveal the surround-attenuation effect when present (Boehler et al., 2009). Subjects performed 10 trial blocks, yielding a total of $80 \mathrm{FP}$ and 80 FO trials at each position.

Assessing the N2pc response. The present experiment is based on an experimental design used in our previous studies on surround attenuation in visual search (Hopf et al., 2006a; Boehler et al., 2009). In those studies, surround attenuation was studied with unilateral search arrays containing one popout target among distractors in the right lower visual quadrant. To assess both the N2pc and surround attenuation at the same time, such unilateral setup could not be used. This is because the N2pc represents a lateralized cortical response, and is derived by comparing the response to a target in the VF contralateral versus ipsilateral to an occipital electrode/sensor. Hence, it is necessary to present search items bilaterally and to direct subjects' attention either to the left or right VF on a given trial. To accommodate this requirement, two search arrays were presented on each trial, with a target popout presented in one, and a potential target popout presented in the opposite VF (Fig. $1 A, B$ ). As the popout colors (red, green) switched VF randomly from trial to trial, and because subjects were instructed to attend to one color (e.g., red) during a given trial block, the focus of attention changed accordingly in a random way from trial to trial. Critically, on a different trial block the same search frames were presented (different randomization), but subjects 
attended to the other color (e.g., green). In other words, attention was directed to different quadrants not by presenting different search frames (with a differing distribution of colored items), but by instructing subjects to attend a different target color. This is important, as this experimental design permitted us to assess the N2pc response without affording low-level stimulation confounds due to color lateralization (Luck, 2005, p.68).

Data recording and analysis. The MEG signal was recorded using a 148-channel BTI Magnes 2500 whole-head magnetometer system (Biomagnetic Technologies), digitized at a rate of $254 \mathrm{~Hz}$, and low-pass filtered from DC to $50 \mathrm{~Hz}$. Eye movements were controlled via a zoom-lens camera and an electrooculogram (EOG) that was recorded simultaneously with the MEG using a Synamps amplifier system (NeuroScan). Both the horizontal and the vertical EOG were recorded with a bipolar montage, using two electrodes behind the lateral orbital angles for the horizontal EOG, and two electrodes below and above the right eye for the vertical EOG. Impedances were kept below $5 \mathrm{k} \Omega$, and an electrode placed at FPZ served as ground. The MEG data were coregistered with anatomical data by digitizing anatomical landmarks [left and right preauricular points, nasion; Polhemus 3Space Fastrak system (Polhemus)], which were then brought into register with magnetic marker fields generated by five spatially distributed coils attached to the subjects' heads.

MEG signals were submitted to online and offline noise reduction (Robinson, 1989). Subsequent artifact rejection was performed by removing MEG epochs exceeding a peak-to-peak threshold of $3 \mathrm{pT}$, and EOG voltage changes exceeding $100 \mu \mathrm{V}$. Epochs containing eye movements, artifacts, or incorrect button presses were excluded from further analysis.

Average event-related magnetic field (ERMF) responses were computed for each individual subject in two different time windows. (1) To characterize the cortical response to the probe, events were averaged time-locked to the probe's onset, and relative to a baseline interval of 400-300 ms preceding it (thus also preceding search-frame onset). Averages were obtained for the different probe-distance conditions (collapsed over positions toward the horizontal and vertical meridian) within both VFs (PD0-PD2 in the left and in the right VF), additionally separated with respect to the distractor conditions (single/multiple) and probe presence (present/absent). To isolate the ERMF response elicited by the probe (probe-related response) from the overlapping response elicited by the search array, FO waveforms were subtracted from FP waveforms (FP-minus-FO difference) of trials with the same target location and distractor condition. This approach has been validated by earlier studies (Luck et al., 1993; Luck and Hillyard, 1995; Vogel et al., 1998) and yields the passive cortical response to the probe under the different experimental conditions. The size of the probe-related response was quantified in each subject, separately for the left and right VF, as mean amplitude of the ERMF difference between the subject's individual efflux and influx maximum, which appeared on average between 120 and 140 ms (Fig. 2A). The time range of analysis was defined relative to the onset of the probe, and was the same for all conditions. The grand average size of the probe-related response as shown in Figure $2 C$ was computed by averaging across subjects' individual differences between efflux and influx maxima before collapsing over responses from left and right VF probes. It should be noted that sensor sites showing efflux and influx maxima varied between subjects (Fig. $2 B$ ), but were very similar for all probe-distance conditions in a given VF and subject. To maximize statistical power, and because the general profile of the probe-related response was found to be very similar in both visual fields, the amplitudes of probe-related response of corresponding probe distances in the left and right VF were averaged.

(2) To characterize the N2pc, the ERMF response to FO-trials was analyzed time-locked to the search frame onset and relative to a $200 \mathrm{~ms}$ baseline directly preceding it. Here, events were averaged separately as a function of the conditions "target VF" (left or right) and "distractor condition" (single or multiple), thus collapsing across all probe-distance conditions. The N2pc was quantified at sensors showing maximum magnetic field effects in corresponding efflux/influx zones (zones representing the same underlying current source in the brain) of both hemispheres. Specifically, the signal of the sensor representing the influx
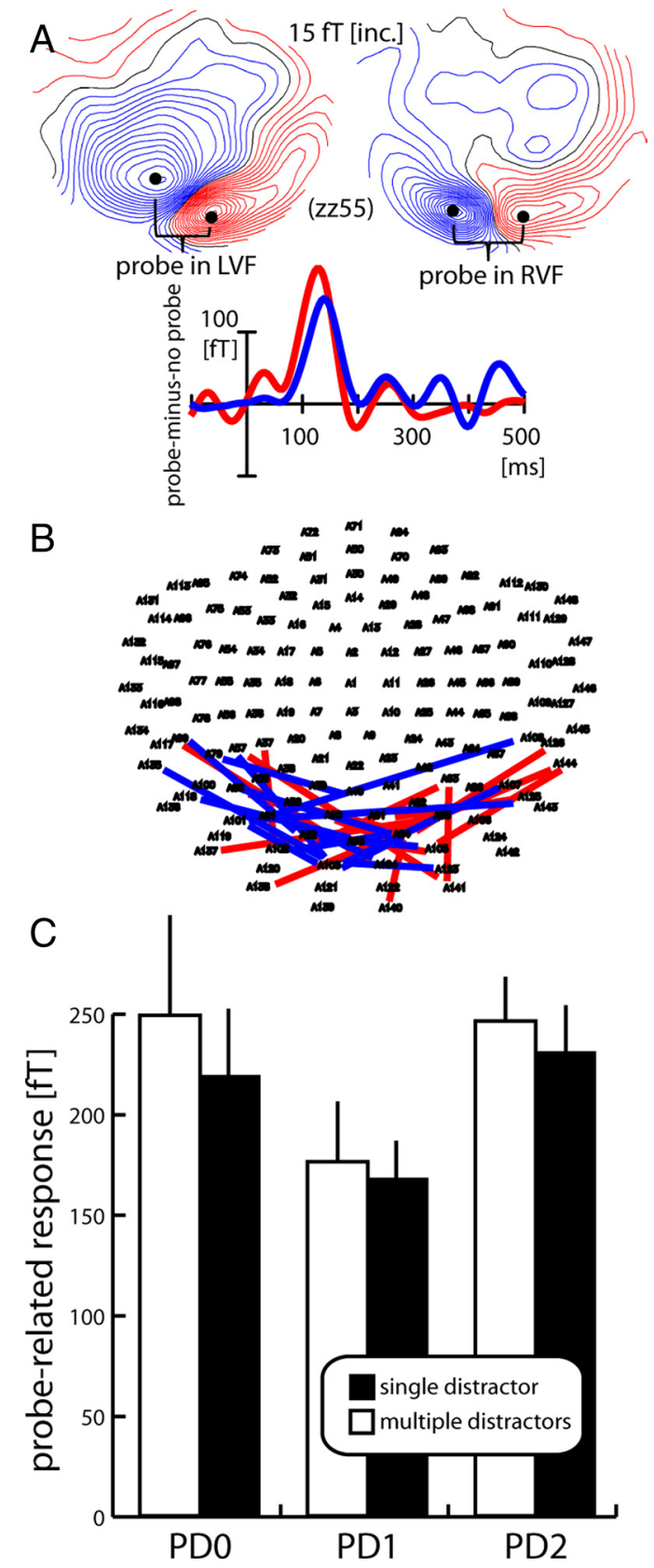

Figure 2. Probe response and surround-attenuation effect (experiment 1). $\boldsymbol{A}$, Mean ERMF response to the probe (FP-minus-F0 difference, average over all probe-distance conditions) in one representative subject (zz55). The maps show the ERMF distribution at $130 \mathrm{~ms}$ after probe onset in the left (left map) and right VF (right map) visible as efflux-influx configurations over central occipital regions, with red lines representing the efflux and blue lines the influx component of the field; the waveforms represent the response to left VF (red trace) and right VF probes (blue trace) collapsed over measurements from sensors (black dots) showing efflux and influx maxima (efflux-minus-influx difference). $\boldsymbol{B}$, Localization of individual maxima of efflux-influx configurations in each subject for probes in the left (red lines) and right (blue lines) VF. Each line connects sensors showing efflux and influx maxima of a given subject. $C$, Grand average (over subjects and VF of probe presentation) probe-related response (FP-minus-F0 difference) as a function of distance to the focus of attention for the single- (black) and multiple- (white) distractor conditions. Error bars show the SEM.

was subtracted from that representing the efflux for all conditions. On the basis of this signal, differences were calculated between the conditions where the target was in the left versus in the right VF.

Statistical data validation was conducted using repeated-measures ANOVAs (rANOVAs). Nonsphericity was corrected based on the Green- 
house-Geisser algorithm (if necessary) with respective results reported with adjusted degrees of freedom.

Current source localization. Current source localization was based on the minimum norm least-squares method (Hämäläinen and Ilmoniemi, 1984; Fuchs et al., 1999) as implemented in the multimodal neuroimaging software Curry 6.0 (Compumedics Neuroscan). Cortical surface constrained current density estimates were computed using realistic models of volume conductor and source compartment. The latter were constructed based on three-dimensional (3D) surface segmentations [boundary element method (Hämäläinen and Sarvas, 1989)] of the CSF space (volume conductor) and the cortical gray matter surface (source compartment) of individual subjects' MR scans (T1-weighted threedimensional spoiled gradient echo sequence; $256 \times 256$ matrix; field of view $25 \times 25 \mathrm{~cm}$; 124 slices; slice thickness $1.5 \mathrm{~mm}$; in plane resolution $0.97 \mathrm{~mm} \times 0.97 \mathrm{~mm}$; echo time $8 \mathrm{~ms}$; repetition time $24 \mathrm{~ms}$; flip angle $30^{\circ}$ ) to constrain source density estimates of individual subjects data [exemplarily illustrated by one representative subject (zz55)]. Segmentation was performed by using the algorithms provided in Curry 6.0. For grand average source density estimates (across subjects), each subject's individual sensor data (sensor coordinates in reference to individual anatomical landmarks; see above, Data recording and analysis) was readjusted to match one subject's set of anatomical landmarks, which served as a reference, and which were then brought into register with a corresponding set of anatomical landmarks of the MNI brain (Montreal Neurological Institute, average of $152 \mathrm{~T} 1$-weighted stereotaxic volumes of the ICBM project, ICBM152). Source density estimates of grand average data (across subjects) were anatomically constrained by 3D surface segmentations of the MNI brain, which served as volume conductor and source compartment.

\section{Experiment 2}

Subjects. Fourteen right-handed subjects took part in the experiment (11 females, mean age: 24.5 ). All participants were neurologically normal students of the Otto-von-Guericke University of Magdeburg and had normal color vision and normal or corrected-to-normal visual acuity. The experiment was undertaken with the understanding and written consent of the subjects and approved by the ethics committee of the Otto-von-Guericke University of Magdeburg. All subjects were paid for participation.

Stimuli and probe procedure. The stimulation protocol of experiment 2 was similar to experiment 1 with the following modifications: (1) Search items and probes were only shown in the lower right visual quadrant. (2) The target was always a more luminant blue C $\left(13 \mathrm{~cd} / \mathrm{m}^{2}\right)$ among less luminant distractor $\mathrm{Cs}\left(1.2 \mathrm{~cd} / \mathrm{m}^{2}\right)$. Note that surround attenuation was shown to be similar under comparable experimental conditions no matter whether the popout target is defined by color or luminance contrast (Hopf et al., 2010). (3) As illustrated in Figure 1, $E$ and $F$, search items appeared at nine possible isoeccentric item locations (PD0 and PD1-PD4 toward the horizontal and vertical meridian) analogous to the experimental setup used in Hopf et al. (2006a). Distance to fixation and item spacing were identical to experiment 1. (4) In experiment 2, the presence/ absence of distractors was varied between separate types of trial blocks. During one type of trial blocks, the target appeared in complete isolation (no-distractor condition), whereas in the other type of trial blocks, the target appeared among eight blue distractors (multiple-distractor condition). Trial blocks with and without distractors alternated throughout the experiment, with subjects performing five blocks with and five blocks without distractors. Each trial block contained 180 trials, resulting in a total of 100 trials per experimental condition and probe distance. In both experimental conditions, the target appeared randomly at one of the nine possible locations, while the probe appeared (on $50 \%$ of the trials) always at the center item position. As in the first experiment, the probe was a small white ring flashed $250 \mathrm{~ms}$ after search frame onset for $50 \mathrm{~ms}$. The experimental procedure was identical to experiment 1 , except that subjects were informed at the start of each trial block about whether distractors would appear or not. Subjects were required to report the orientation of the target gap (left/right) with a two-alternative button press of the right hand index/ middle finger.

Data recording and analysis. Data recording was identical to experiment 1 . For data analysis, average ERMF responses were computed for the different probe distances (collapsed over positions toward the horizontal and vertical meridian) and distractor conditions. In addition, averages were obtained separately for FP and FO trials. As in the first experiment, the ERMF response elicited by the probe proper (proberelated response) was isolated from the overlapping response elicited by the search array by subtracting FO waveforms from corresponding FP waveforms (trials corresponding regarding target location and distractor condition). The size of the probe-related response was quantified in each subject, as the mean amplitude of the ERMF difference between the efflux and influx maximum appearing between 120 and $140 \mathrm{~ms}$.

\section{Results}

Experiment 1

Behavioral performance

Subjects committed slightly more errors when many distractors were present (mean proportion of errors in multiple-distractor condition: $7.9 \%$, in single-distractor condition: $5 \%, F_{(1,14)}=$ $27.8, p<0.001)$. Furthermore, multiple distractors led to longer response times (mean response time in multiple-distractor condition: $565 \mathrm{~ms}$, in single-distractor condition: $540 \mathrm{~ms}, F_{(1,14)}=$ $67.2, p<0.001)$. Response time was generally faster in the right than the left VF (mean response time in right VF: $545 \mathrm{~ms}$, in left VF: $\left.557 \mathrm{~ms}, F_{(1,14)}=5.4, p<0.05\right)$, but this lateralization was uninfluenced by the number of distractors $(p>0.25)$. Response accuracy was not lateralized, nor was there an accuracy $\times$ VF interaction (both $p>0.25$ ).

\section{MEG results: surround attenuation}

The effect of surround attenuation was assessed by analyzing the size of the cortical response to the probe stimulus as a function of its distance from the focus of attention. To obtain the proberelated response, it was necessary to separate the response to the probe from overlapping cortical activity elicited by the search frame proper. To this end, activity elicited by search frames without a probe was subtracted from search frames followed by a probe (FP-minus-FO difference). Figure $2 \mathrm{~A}$ displays the time course and field distribution of the overall response to probes in the left (red trace and left map) and right (blue trace and right map; data collapsed over probe-distance and distractor conditions) VF of one representative single subject (zz55). As in our previous studies (Hopf et al., 2006a; Boehler et al., 2009), the probe-related response appears as a simple efflux-influx configuration (brackets) over the occipital cortex, consistent with a current origin in early visual cortex. Figure $2 B$ summarizes the localization of efflux-influx configurations of all subjects with each colored line connecting corresponding efflux and influx maxima of a given subject for LVF probes (red lines) and RVF probes (blue lines). The bar graph in Figure $2 C$ shows the size of the probe-related response as a function of the probe's distance to the focus of attention (data collapsed for probes in the left and right VF). The bars represent the mean ERMF response of the single- (black) and the multiple- (white) distractor conditions between 120 and $140 \mathrm{~ms}$ after probe onset averaged over measurements from each subject's individual efflux-influx sensor maximum (efflux-minus-influx difference) as shown in Figure $2 B$. Apparently, the probe-related response is reduced when the position next to the probe is attended (PD1) relative to when attention is focused onto the probe's location (PD0) or two items away from the probe (PD2). This activity pattern clearly replicates our previous observations of a zone of attenuated excitability surrounding the focus of attention-the surround-attenuation effect. Importantly, surround attenuation is present under both the multiple- and the single-distractor conditions. Responses were overall somewhat smaller for the single-distractor condition, but 
there is little difference regarding the surround-attenuation effect.

It should be noted that the present results do not rule out that there may be subtle differences of the spatial profile of the focus of attention between the multiple- and the single-distractor conditions. It is possible that changes of the width of surround attenuation appeared at a scale for which item spacing in the present experiment, that is, the spatial sampling of the profile, was not dense enough. However, such small differences are not critical, since the important demonstration here is that surround attenuation appears regardless of whether distractors are present or absent in the target's surround. To validate the surroundattenuation effect statistically, a two-way rANOVA with the factors probe distance (PD0/PD1/PD2) and number of distractors (multiple/single) was computed. This analysis yielded a significant main effect of probe distance $\left(F_{(1.8,25)}=3.7, p<0.05\right)$, validating the presence of surround attenuation in both conditions, but there was no significant effect of number of distractors $(p>0.4)$, nor was there an interaction between both factors $(p>0.4)$. Subsequent pairwise comparisons (PD0 vs PD1 and PD1 vs PD2) yielded results compatible with surround attenuation. Significant main effects of probe distance were observed for the relevant comparisons (PD0 vs PD1: $F_{(1,14)}=5.3, p=0.037$; PD1 vs PD2 $\left.F_{(1,14)}=8.1, p=0.013\right)$, while there was no effect of number of distractors or an interaction of number of distractors $\times$ probe distance (all $p>0.25$ ).

The surround attenuation effect is further illustrated in Figure 3 , which shows the distribution of attenuated cortical excitability at PD1 for the single- and multiple-distractor conditions of the grand average across subjects (Fig. $3 A$ ) and a selected individual subject (zz55) (Fig. 3B). Attenuation of activity at PD1 was assessed relative to the activity level at PD2. That is, current density estimates (see Materials and Methods) of the PD2-minus-PD1 ERMF difference were computed for probes in the left (left column) and right (right column) VF. To indicate that the activity maps represent the distribution of attenuated activity, current density estimates are shown as negative values with white representing the strongest attenuation. Obviously, attenuation is distributed over the occipital cortex with a maximum over early visual areas contralateral to the VF of probe presentation (black outlines). The fact that the attenuation is maximal in early visual cortex areas is particularly apparent in the source localization analysis of the single-subject data, which shows maximum current density estimates in a region consistent with the primary visual cortex and estimates of lower strength in extrastriate regions. This distributional pattern is clearly consistent with our previous analysis of the cortical locus of surround attenuation, which revealed maximum attenuation effects in early-tointermediate level areas of visual cortex (Hopf et al., 2006a). Importantly, the distribution and strength of attenuation is very similar when comparing the single- and multipledistractor conditions, again indicating that the occurrence of surround attenuation is rather uninfluenced by the presence/ absence of distractors.

\section{N2pc response}

Figure $4 A$ shows waveforms and ERMF distribution of the overall N2pc response (grand average across subjects, collapsed over the single- and multiple-distractor conditions) together with current source localization results of the grand average $\mathrm{N} 2 \mathrm{pc}$ and the $\mathrm{N} 2 \mathrm{pc}$ response of a representative single subject (zz55). In the waveforms, the N2pc is visible as left (solid trace) versus right (dashed trace) VF target difference between 200 and 330 ms after
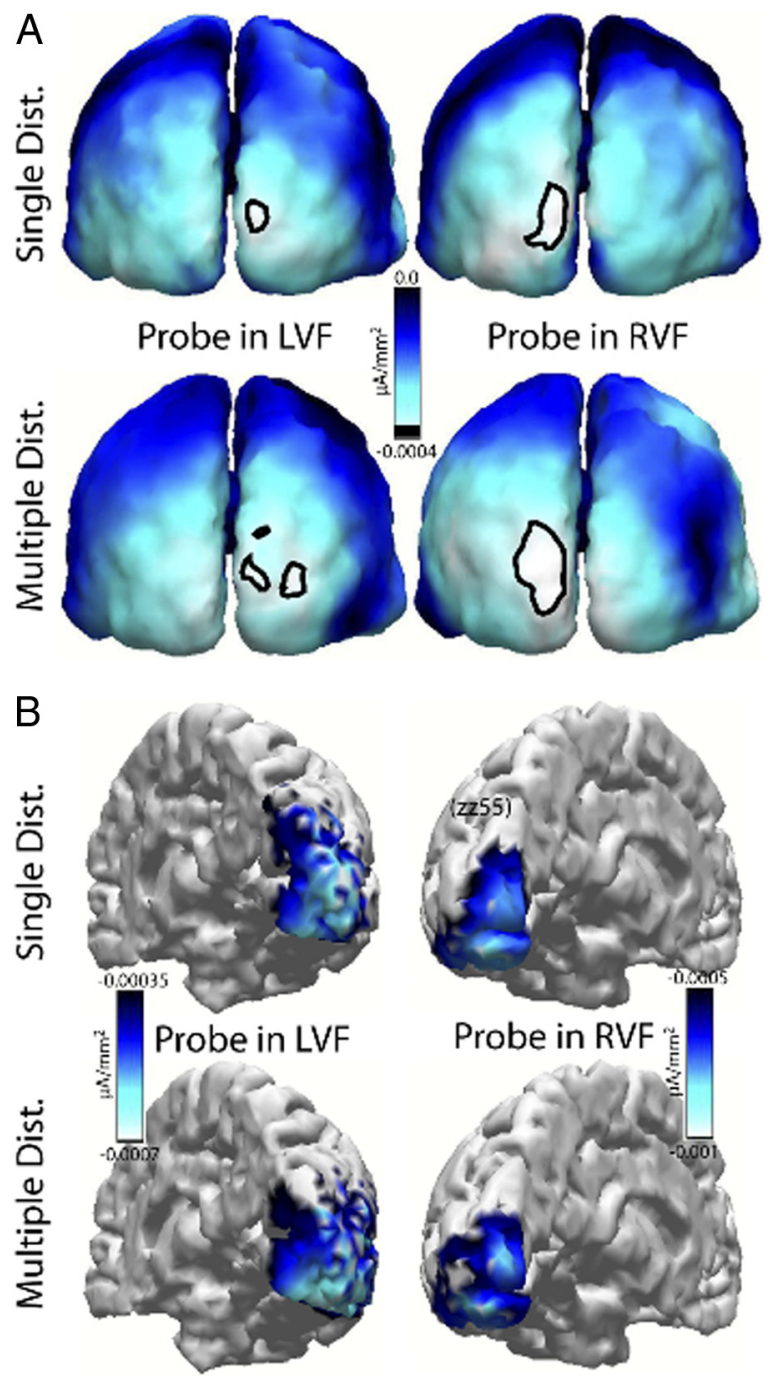

Figure 3. Source density analysis of the surround-attenuation effect (experiment 1). Cortical distribution of the surround-attenuation effect (PD2-minus-PD1 ERMF difference) of the singleand multiple-distractor conditions revealed by probes presented in the left (left column) and the right (right column) VF. $A$, Current source density estimates of the grand average (over subjects) attenuation of the probe-related response. The outlines index the attenuation maxima at the threshold marked by the black line in the scale bar. $\boldsymbol{B}$, Source density estimates of the surround attenuation effect in a single representative subject (zz55). A negative scale was chosen to highlight that the maps show the distribution of attenuated responses.

search-frame onset (gray area between traces). Note that the waveforms reflect activity measured at, and collapsed over, sensors at efflux and influx maxima (black dots). Collapsing was done by subtracting influx from efflux activity. The map shows the distribution of the left minus right VF target difference at 270 $\mathrm{ms}$ in which the N2pc appears as efflux-influx configuration over left and right inferior occipitotemporal regions (ellipses). Red field lines reflect the efflux and blue lines the influx component. Current sources underlying the N2pc are expected to appear in the transition zone between efflux and influx maxima, which is confirmed by current source estimates of the grand average N2pc (gav) as well as the N2pc of an individual subject (zz55) shown on the right [see Hopf et al. (2000) for a detailed discussion of the magnetic field distribution underlying the magnetic analog of the $\mathrm{N} 2 \mathrm{pc}$. Current maxima appear in inferior occipitotemporal regions of the left and right visual cortex. Those localizations are perfectly in line with our previous observations (Hopf et al., 2000, 

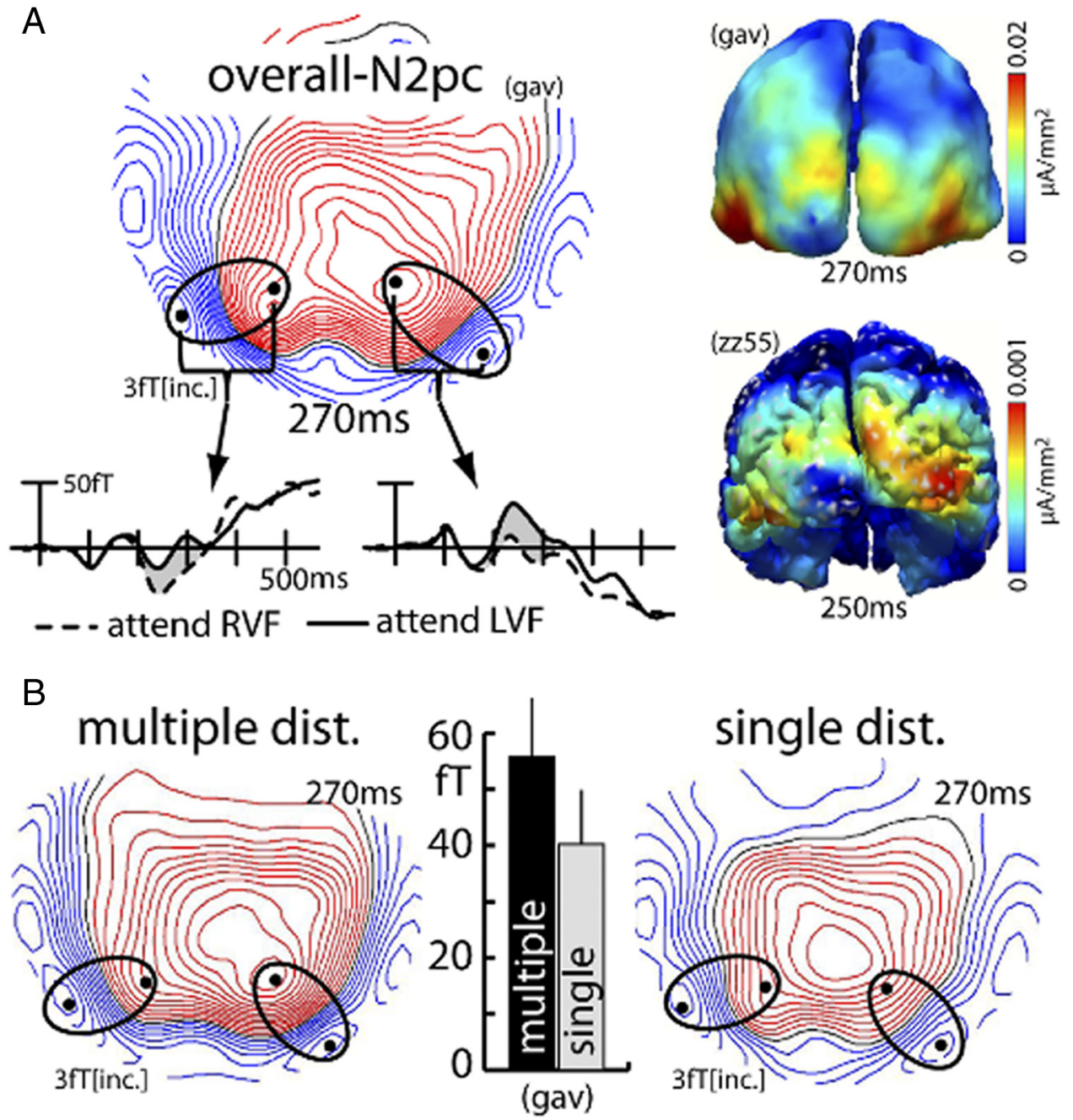

single dist.

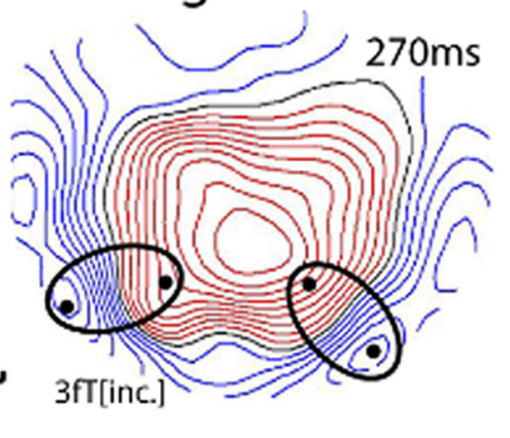

Figure 4. N2pc effect (experiment 1). A, Waveforms, ERMF distribution, and current source density estimates of the overall N2pc [grand average (gav) over subjects; collapsed over distractor conditions; F0-trials only]. The waveforms show the response recorded at sensor sites (black dots) positioned over maxima of the efflux-influx configurations. The response was collapsed over measurements from sensors (black dots) representing corresponding efflux and influx maxima (efflux-minus-influx difference). The N2pc effect is visible as the difference between waveforms (gray area) elicited by targets in the left (solid traces) and right (dashed traces) VF between $\sim 200$ and $330 \mathrm{~ms}$ after search frame onset. The activation maps on the right show the current source density distribution of the N2pc of the grand average (top) and of a single representative subject (zz55, bottom). $\boldsymbol{B}$, Field distribution of the multiple- (top) and single- (bottom) distractor conditions of the grand average N2pc at $270 \mathrm{~ms}$. The bar graph shows the amplitude of the grand average (gav) N2pc response separately for the two distractor conditions (collapsed over left and right hemisphere responses).

2002, 2006b). Figure $4 B$ shows the grand average N2pc field distribution at $270 \mathrm{~ms}$ separately for the multiple- (left) and the single- (right) distractor conditions. A comparison of the maps indicates that both conditions produce a prominent $\mathrm{N} 2 \mathrm{pc}$ with a similar field distribution. The size of the N2pc effect, however, is larger in the multiple-distractor condition. This difference in amplitude is further illustrated by the bar graph showing the amplitude of the N2pc of the multiple- (black) and single- (gray) distractor condition for the grand average. Measurements of the grand average $\mathrm{N} 2 \mathrm{pc}$ were taken from sensor sites highlighted by the black dots inside the ellipses (efflux-minus-influx difference) and then collapsed over left and right hemisphere effects. Figure $5 \mathrm{~A}$ illustrates a further notable observation. Not only is the amplitude of the N2pc larger for the multiple-distractor condition, around $290 \mathrm{~ms}$ its field distribution also changes to a more posterior region in the left occipitotemporal cortex (arrow, dashed ellipses indicate the maximum field effect at $270 \mathrm{~ms}$ shown in Fig. $4 B$ ). This suggests that at $290 \mathrm{~ms}$ the N2pc of the multipledistractor condition arises from an earlier (i.e., hierarchically lower) visual cortex area than around 270 ms. Note that such change in the locus of source activity was seen in previous analyses of the N2pc (Hopf et al., 2006b) when search required an increase of the resolution of attention. Apparently, no such change of the field distribution appears for the single-distractor condition.

Figure $5 B$ provides a further analysis of this change in field distribution. Shown are source density estimates of the overall $\mathrm{N} 2 \mathrm{pc}$ at $270 \mathrm{~ms}$ (top) together with the multiple- minus singledistractor difference of the N2pc at $290 \mathrm{~ms}$ (bottom). Traces in the middle show the time course of (normalized) source activity at the current density maxima of the overall N2pc (dashed) and the multiple-minus-single N2pc difference (solid). Obviously, the distribution of the multiple-minus-single-distractor N2pc difference displays current source activity more posterior than the maximum of the overall N2pc at 270 ms (dashed outline). The current source localization analysis confirms the impression from the field distributions in Figure 5A, namely that multiple distractors in the target's surround produce additional N2pc ac- 
A
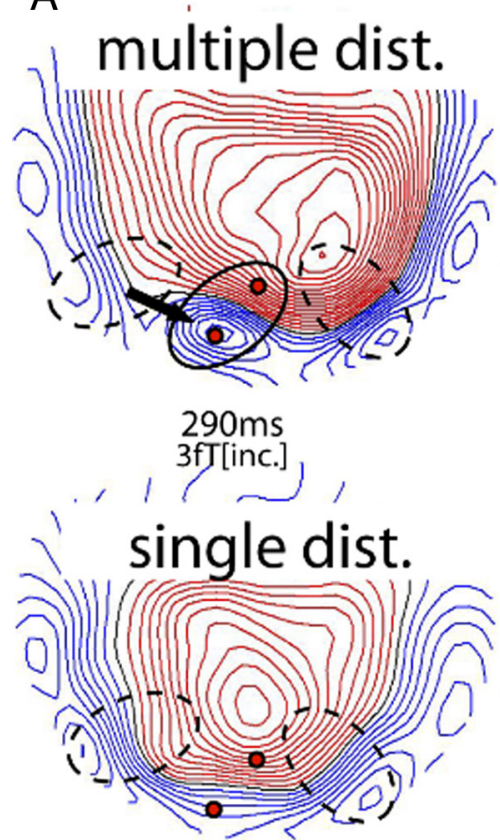

B

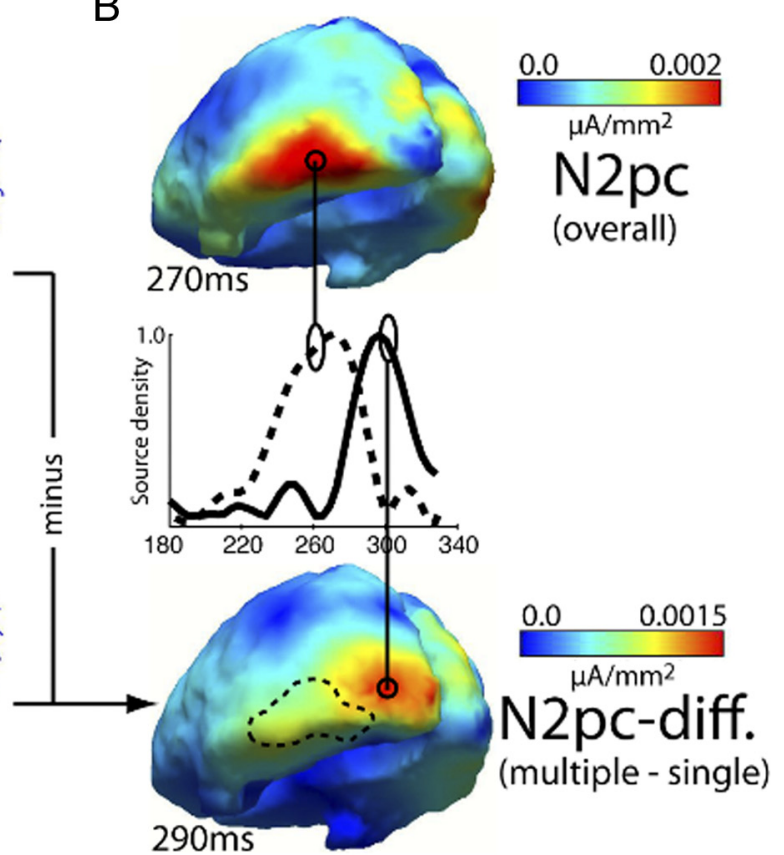

Figure 5. ERMF distribution underlying the N2pc at $290 \mathrm{~ms}$ (experiment 1). $\boldsymbol{A}$, ERMF distribution of the N2pc (average over subjects) of the multiple- (top) and single- (bottom) distractor conditions $290 \mathrm{~ms}$ after search frame onset. The multiple-distractor condition shows an additional posterior occipital effect (solid ellipse) not present in the single-distractor condition. Field effects underlying the N2pc at $270 \mathrm{~ms}$ (compare Fig. 4) are marked by dashed ellipses. B, Current density distribution of the overall N2pc (top) in comparison to estimates computed based on the N2pc difference between the multiple- and the single-distractor conditions (estimates from the average ERMF response over subjects). The traces in the middle show the normalized time course of source activity obtained from the indicated locations (circles). The dashed contour in the lower map shows the distribution of the overall N2pc source activity at 270 ms as a comparison.

tivity in more posterior, presumably hierarchically lower levels of the visual cortex.

For statistical validation, the N2pc effect was quantified as the mean ERMF waveform difference (left-minus-right VF target response) between 200 and $330 \mathrm{~ms}$. Data were obtained from occipital sensors showing maximal efflux and influx in the left and the right hemisphere (Fig. $4 B$, black dots). To simplify the analysis, in each hemisphere responses from sensors showing corresponding efflux and influx components were combined by subtracting the influx from the efflux maximum. Moreover, the $\mathrm{N} 2 \mathrm{pc}$ was derived on each side by subtracting conditions where attention was focused on the right from the corresponding conditions with attention on the left. A two-way rANOVA on these $\mathrm{N} 2 \mathrm{pc}$ amplitudes with factors number of distractors (multiple/ single) and sensor hemisphere (left/right) was computed, which yielded a significant effect of number of distractors $\left(F_{(1,14)}=\right.$ 8.03, $p<0.05$ ), confirming that the N2pc was larger for the multiple-distractor condition. There was, however, no significant effect of sensor hemisphere $\left(F_{(1,14)}=2.91, p=0.11\right)$, and no significant interaction between the two factors $\left(F_{(1,14)}=2.6, p=\right.$ $0.13)$. A separate statistical analysis was performed to validate the change of field distribution of the N2pc toward more posterior sensor sites in the multiple-distractor condition. For this analysis, a one-way rANOVA with the factor number of distractors (multiple/single) was computed based on the mean field response between 280 and $330 \mathrm{~ms}$ at more posterior sensor sites highlighted in red in Figure $5 \mathrm{~A}$. A significant effect of number of distractors $\left(F_{(1,14)}=10.5, p<0.01\right)$ was observed, confirming that the N2pc of the multiple-distractor condition shows an additional response over a more posterior occipitotemporal region in the left hemisphere.

\section{Experiment 2}

Experiment 1 shows that eliminating distractors, except for one presented opposite to the target, did not eliminate the surroundattenuation effect. In fact, the size of surround attenuation remained almost unchanged, speaking against surround attenuation arising from a neural operation that directly serves distractor attenuation. However, one may envision distractor attenuation to be an all-or-none operation, appearing regardless of the number and location of distractors. It is therefore possible that in experiment 1 the one distractor opposite to the target triggered distractor attenuation in such all-or-none manner, thereby producing surround attenuation to the same amount as in the multiple-distractor condition. Hence, it remains to be demonstrated that surround attenuation arises even when distractors are completely eliminated from the search frame. Furthermore, distractor suppression has been shown to be contingent on the expectation of distractors appearing at preknown locations (Awh et al., 2003; Serences et al., 2004). Multiple- and single-distractor trials were randomly mixed in experiment 1, presumably leading subjects to build an overall expectation that distractors appear on every upcoming trial. If surround attenuation reflects such expectation-related downmodulation of the neural responsivity at potential distractor locations, surround attenuation in the single-distractor condition would still reflect a consequence of distractor processing. It is therefore critical to assess whether surround attenuation also arises when subjects do not expect distractors to appear. Experiment 2 addressed both issues by (1) completely eliminating distractors on $50 \%$ of the trials and (2) presenting search frames with and without distractors in separate trial blocks, such that 
A
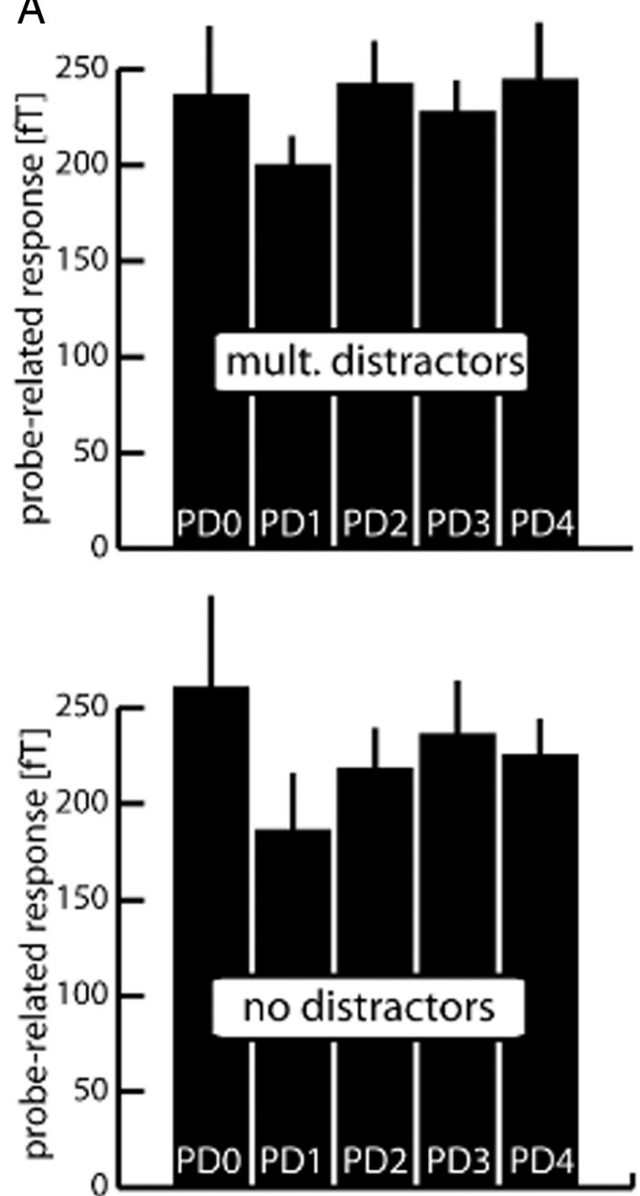

B

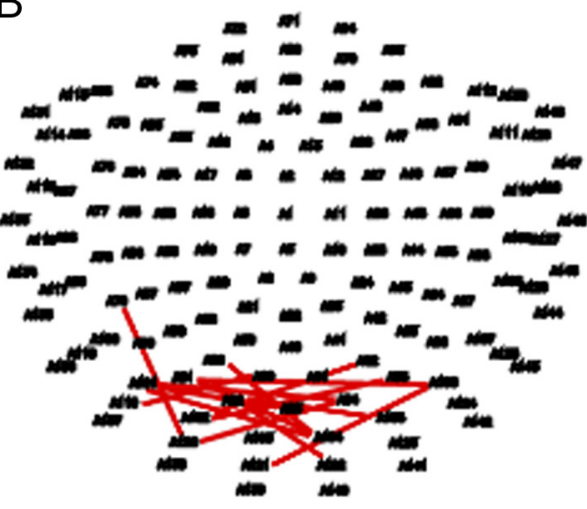

C

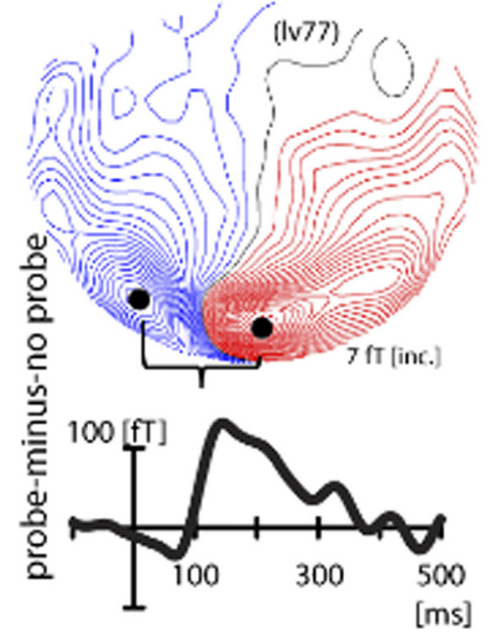

Figure 6. Probe response and surround-attenuation effect (experiment 2). $\boldsymbol{A}$, Average probe response (over subjects) as a function of distance to the focus of attention (PD0 -PD4) for the multiple-distractor (top) and the no-distractor (bottom) conditions. The bar graphs represent the average ERMF response between 120 and $140 \mathrm{~ms}$ after probe onset. Equivalent probe distances toward the horizontal and vertical meridian were collapsed. Before averaging over subjects, the size of the probe-related response was determined in each subject by measuring the amplitude difference between the individual efflux and influx maxima representing the probe-related response. $\boldsymbol{B}$, Localization of efflux-influx maxima of each subject $(n=14)$ participating in experiment 2. Each red line connects the efflux and influx maximum of the probe-related response of one subject. C, Probe-related ERMF response (FP-minus-F0 difference averaged over all probe-distance conditions) of a single representative subject (Iv77).

during blocks of no-distractor trials subjects had no reason to expect distractors at nontarget locations.

\section{Behavioral performance}

Analogous to the first experiment, subjects committed slightly more errors when distractors were present versus absent (present: $2.3 \%$ vs absent: $1.7 \%$ ), but this difference did not reach statistical significance $\left(F_{(1,13)}=3.37, p<0.09\right)$. In contrast, the presence of distractors led to a significant increase in response time (present: $510 \mathrm{~ms}$ vs absent: $471 \mathrm{~ms} ; F_{(1,13)}=50.3, p<$ $0.0001)$.

\section{MEG results}

Figure $6 \mathrm{~A}$ shows the size of the probe-related response (FPminus-FO difference) as a function of the probe's distance to the focus of attention (data collapsed over equivalent probe distances toward the horizontal and vertical meridian). The bars represent the grand average (over subjects) response of the multiple-distractor (top) and the no-distractor condition (bottom) between 120 and $140 \mathrm{~ms}$ after probe onset. The grand average was obtained by averaging the probe-related response measured at the subjects' individual sensors showing maximum efflux and influx effects (efflux-minus-influx difference). Respective sensors are summarized in Figure $6 B$, with each line connecting efflux and influx maxima of one subject. Figure $6 C$ shows the waveform and field distribution the probe-related response (collapsed over probe distances and distractor conditions) of a typical subject (lv77). As visible in Figure $6 A$, there is a reduced response at PD1 relative to $\mathrm{PD} 0$ and relative to PD2-PD4 in both conditions, indicating that surround attenuation is present no matter whether distractors are present or completely absent from the search display. A rANOVA with the factors probe distance (PD0-PD4) and distractor presence (absent/present) confirms the validity of this observation by yielding a significant main effect of probe distance $\left(F_{(1,13)}=6.03, p<0.01\right)$ but no main effect of distractor presence $\left(F_{(1,13)}=0.09\right)$, and also no probe distance $\times$ distractor presence interaction $\left(F_{(1,13)}=0.37\right)$. Subsequent pairwise comparisons revealed that the significant effect of probe distance is due to PD1 being significantly smaller than PD0 ( $p<0.05)$, as well as being smaller than all farther away probe distances (PD1 vs PD2: $p<0.005$; PD1 vs PD3: $p<0.05$; PD1 vs PD4: $p<0.005)$. All pairwise comparisons not involving PD1 were not significant (all $p>0.5$ ). When comparing the responses to PD0 and PD1 between distractor conditions in Figure 6, it appears that the attenuation at PD1 is stronger in the no-distractor condition. However, a 
planned rANOVA with the factors probe distance (PD0/PD1) and distractor presence (present/absent) only revealed a significant main effect of probe distance $\left(F_{(1,13)}=4.8, p<0.05\right)$, not a probe distance $\times$ distractor presence interaction $\left(F_{(1,13)}=0.5\right)$, indicating that this difference is not statistically significant.

\section{Discussion}

Three important observations were made in the reported experiments. (1) Surround attenuation was observed with similar effect size and cortical distribution no matter whether the target was surrounded by distractors or not. (2) Surround attenuation was seen even when subjects did not expect distractors at any location because they were never presented on the trials of a complete experimental block. (3) The N2pc, in contrast, increased in amplitude when distractors appeared in the target's surround. The first two observations are notable in view of our previous interpretations, where we argued that surround attenuation may be optimal for eliminating distractor interference in the vicinity of the target (Hopf et al., 2006a). The present results speak against this interpretation. Furthermore, experiment 2 rules out the further possibility that surround attenuation in the single-distractor condition of experiment 1 reflects anticipatory suppression of item locations that were empty but expected to contain distractors (Awh et al., 2003; Serences et al., 2004). While those observations may appear unexpected, they complement our recent experimental findings (Boehler et al., 2009) showing that the discrimination of a simple color popout among distractors did not produce surround attenuation. Conversely, search requiring spatial scrutiny for discriminating the same popout target (gap-orientation discrimination) was associated with surround attenuation. We concluded that it was the requirement to discriminate details of the target with high spatial resolution that produced surround attenuation. The fact that the gapdiscrimination task of the present experiments required the same degree of spatial resolution, regardless of whether distractors surrounded the target or not, is consistent with surround attenuation being a consequence of increasing the spatial resolution of attention. The present observations provide an important qualification, namely, that distractor suppression and increasing of the spatial resolution of attention are subserved by separable operations.

How surround attenuation links with increasing the spatial resolution of discrimination can be reconciled in the framework of the selective tuning (ST) model of visual attention (Tsotsos et al., 1995, 2008), and it turns out that the dissociation between surround attenuation and distractor processing can also. A key notion of many theories of attentional selection, originating with $\mathrm{ST}$, is that focusing of attention arises from top-down selection in visual cortex (Lamme and Roelfsema, 2000; Deco and Zihl, 2001; Hochstein and Ahissar, 2002; Spratling and Johnson, 2004). Neural evidence supporting this proposal is mounting (Mehta et al., 2000; Martínez et al., 2001; Noesselt et al., 2002; Buffalo et al., 2010). Importantly, and differing from the other models, in ST top-down selection produces surround attenuation as an inherent consequence for which the presence of distractors is not critical. Figure $7 A-D$ provides an illustration. Shown is a sequence of processing steps of two input items, representing a target (red) and a distractor (blue). Figure $7 A$ illustrates the activation in visual cortex after the initial feedforward sweep of processing has reached the top level $(n)$ of a four-level visual cortical hierarchy. The forward activation is represented by diverging pyramids of red and blue units that show considerable overlap. The recurrent activation begins with a competitive selection mechanism [real- ized via a winner-take-all (WTA) computation] to determine the unit representing the target at the top level. Selection among item representations always involves competition, and the greater the ambiguity created by overlapping representations - maxima of red and blue units (double arrow) - the greater the competition. At this stage attention is unfocused, as its resolution corresponds to the full receptive field of the unit involved, i.e., the extension of the downward diverging pyramid (dashed triangle in Fig. 7A). If higher spatial resolution is required, further top-down processing is involved (Fig. $7 B-D$ ), in the form of a backward-tracing WTA process that propagates downward from level to level through the cortical hierarchy, thereby eliminating forward projections from units not representing the target at a given level (Fig. 7D). This leads to attenuation around a narrowing pass zone of nonattenuated forward connections-the surround-attenuation effect. Importantly, surround attenuation will not reflect the number of distractors, because which connections are eliminated depends on whether they are contained in the zone of forward convergence from one level to the winner at the next higher level, independent of whether they represent input from distractors (as at $n-1$ in Fig. $7 B$ ) or not (as at level $n-2$ in Fig. 7C).

The N2pc has been suggested to reflect neural processes that serve to filter confounding effects of distractors onto target selection (Luck and Hillyard, 1994; Eimer, 1996; Luck et al., 1997; Hopf et al., 2002; Hickey et al., 2009). Consistently, cortical processing subserving distractor attenuation was enhanced in the multiple-distractor condition. Neural activity underlying the $\mathrm{N} 2 \mathrm{pc}$, however, does not overlap with activity underlying surround attenuation. This dissociation is further suggested by the fact that surround attenuation and the N2pc differ in their locus of maximum cortical expression. In line with previous observations (Hopf et al., 2000), the N2pc shows source maxima in ventral occipitotemporal cortex. The reduction of source activity reflecting surround attenuation, in contrast, increases toward early occipital cortex regions.

But how are both signatures related? Considering the functional significance of the N2pc put forward by the ambiguity resolution theory (Luck et al., 1997) in the framework of the ST model provides a possible explanation and reconciles three important observations reported here.

1. Following the ambiguity resolution theory (Luck et al., 1997), a parsimonious hypothesis would be that the N2pc reflects competition among potential winners at a given hierarchical level, with stronger competition between units leading to larger N2pc amplitudes. Let the situation in Figure $7 A$ reflect the target (red) and one nearby distractor (blue) of the multiple-distractor condition. At the top level $(n)$, pyramids show considerable overlap. The fact that the subject is instructed to search for a red target leads to a bias in favor of the red units-and the resulting competition is reflected by the N2pc response. At level $n-1$, the pyramids still overlap, although to a lesser extent, and again, the biasing of competition in favor of red units that affects the whole pyramid plays a role (double arrow in Fig. $7 B$ ) in determining the winner. Now, imagine separating the red and blue input by many units, as in our single-distractor condition. Then, the forward pyramids would not overlap at level $n-1$, and although the bias is still present due to the subject's instruction, competition between red and blue is not present among $(n-1)$-level units that project forward to the global winner. Consequently, the size of the N2pc response would be reduced because the amount of competition in the top-down pyramid defined by the global winner is reduced. 


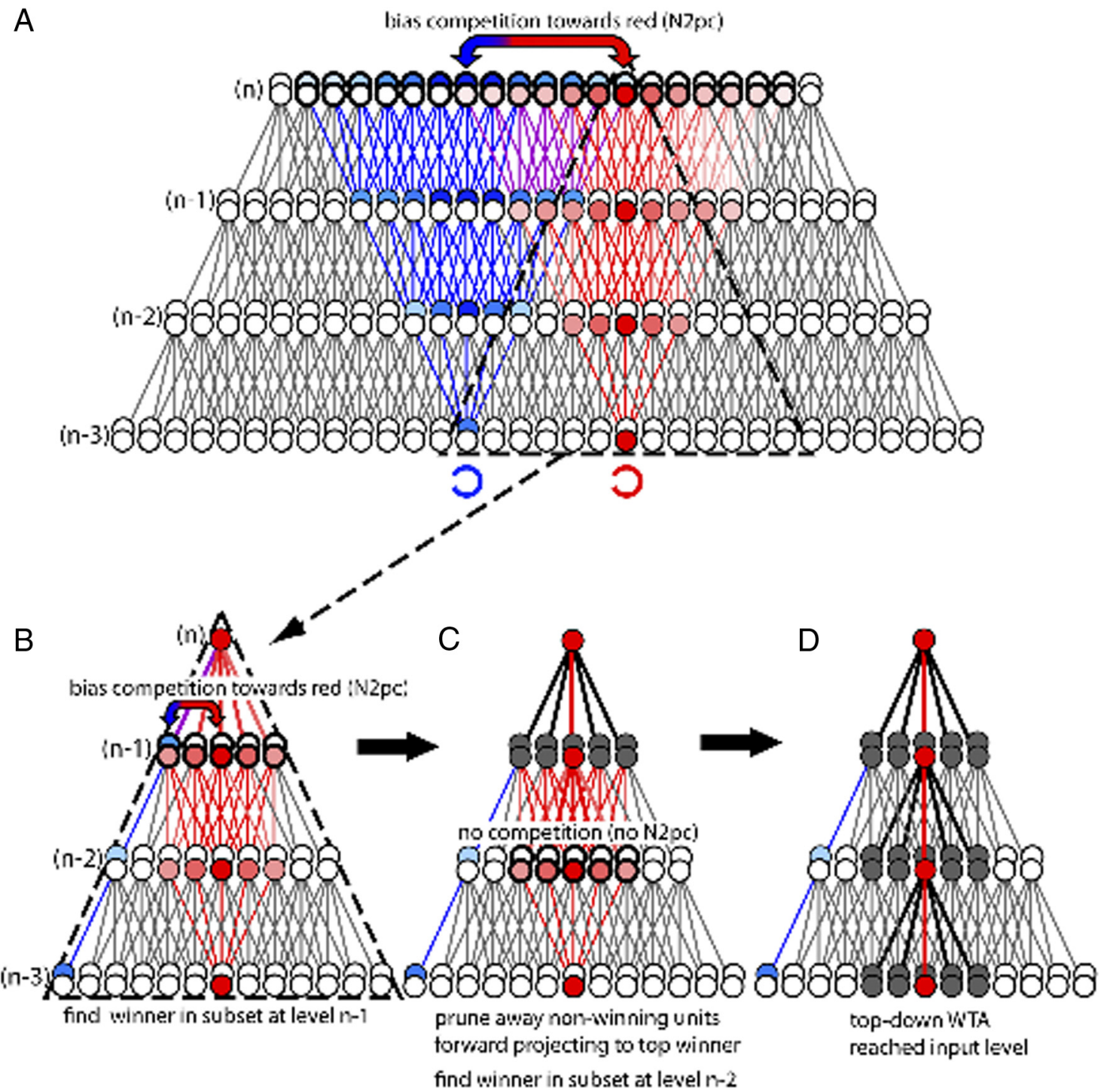

Figure 7. Linking the N2pc and surround attenuation in the ST model of visual attention. $A$, Illustration of the feedforward activation pattern elicited by two search items (a red target and a blue distractor C) in a four-level hierarchical connectivity model of the retinotopic visual cortex. Feedforward activations are represented by diverging pyramids (fan-out factor of 5:1) of red and blue units, which show increasing overlap toward the top level $(n)$ associated with an increase of competition among units representing the target and the distractor. To find a global winner representing the target at the top level (the dark red unit), top-down attention must decide the competition in favor of the red units-an operation proposed to be indexed by the N2pc (double arrow). This sets the starting point for a top-down propagating WTA process to increase the spatial resolution of discrimination beyond that of the top-level units. $B$, Downward divergent connectivity associated with the top-level winner (units within dashed pyramid in $A$ ) determines the extension of the WTA process at the next lower level $(n-1)$. At $n-1$, a new winner is determined (dark red) and connections from nonwinning units to the next level winner $(n)$ are pruned (black lines and gray units in $\boldsymbol{C}$. This process propagates downwards until the input level $(n-3)$ is reached (D), thereby leaving a pass zone matching the spatial resolution of units at the input level surrounded by attenuation (surround attenuation). Note that at level $n-1$, the forward projection from blue units still overlaps with units representing the red input, which gives rise to competition (double arrow in $\boldsymbol{B}$ ) and therefore modulatory effects producing the N2pc. In contrast, at level $n-2$, red-representing units projecting to the winner at level $n-1$ do not overlap with blue units. Hence, no competition arises and no N2pc response appears.

2. This interpretation further predicts that distractor interference at smaller spatial separations (as in the multipledistractor condition) produces N2pc activity at lower levels in the visual processing hierarchy. Evidence in line with this prediction has been provided recently (Hopf et al., 2006b). Competition due to item separation and associated attention effects were shown to scale with receptive field (RF) size and the corresponding hierarchical level of cortical representation (Kastner et al., 2001; Bles et al., 2006). The present data add further support. Source activity underlying the N2pc showed an additional more posterior occipitotemporal maximum around $290 \mathrm{~ms}$ (Fig. 5) in the multiple- but not the singledistractor condition. This suggests an activity origin in a hierarchically lower-level visual cortex region, where competition can be decided at a smaller spatial scale of item separation. Note that this more posterior source activity was only observed in the left hemisphere, which is somewhat unexpected. However, scale-dependent lateralization of processing in ventral extrastriate cortex has been reported previously. The selection of local detail in hierarchical patterns revealed activity lateralized to the left extrastriate cortex (Heinze and Münte, 1993; Fink et al., 1996; Han et al., 2002). It is possible that the present lateralization reflects such left-hemisphere preference for local detail.

3. Finally, competition among item representations generally increases toward higher levels of the visual cortical hierarchy. An attentional operation reflecting the resolution of competition among potential winners, like the $\mathrm{N} 2 \mathrm{pc}$, is there- 
fore expected to arise in cortical regions representing higher levels of the hierarchy. The neuromagnetic signature of surround attenuation, in contrast, is expected to increase toward lower levels of the hierarchy, because the number of units excluded by the top-down pruning process increases with decreasing levels in the hierarchy. Hence, consistent with the present observations, the locus of effect underlying the N2pc and surround attenuation dissociate toward opposite directions in the visual hierarchy.

To summarize, the reported experiments show that cortical activity underlying surround attenuation and the N2pc reflect distinct operations of neural attenuation. The former-a consequence of increasing the resolution of attention-is independent of the presence/absence of distractors. The latter, known to reflect distractor competition, increases when adding distractors. While reflecting different operations, we suggest that both are linked during top-down attentional selection in visual cortex. In line with previous notions (Luck et al., 1997) the N2pc may reflect the selection of a winner among competing item representations at hierarchical levels where large RF sizes give rise to competition; surround attenuation may reflect the subsequent spatial demarcation of the winning representation as suggested by the ST model.

\section{References}

Awh E, Matsukura M, Serences JT (2003) Top-down control over biased competition during covert spatial orienting. J Exp Psychol Hum Percept Perform 29:52-63.

Bles M, Schwarzbach J, De Weerd P, Goebel R, Jansma BM (2006) Receptive field size-dependent attention effects in simultaneously presented stimulus displays. Neuroimage 30:506-511.

Boehler CN, Tsotsos JK, Schoenfeld MA, Heinze H-J, Hopf JM (2009) The center-surround profile of the focus of attention arises from recurrent processing in visual cortex. Cereb Cortex 19:982-991.

Buffalo EA, Fries P, Landman R, Liang H, Desimone R (2010) A backward progression of attentional effects in the ventral stream. Proc Natl Acad Sci U S A 107:361-365.

Chelazzi L, Miller EK, Duncan J, Desimone R (1993) A neural basis for visual search in inferior temporal cortex. Nature 363:345-347.

Chelazzi L, Duncan J, Miller EK, Desimone R (1998) Response of neurons in inferior temporal cortex during memory-guided visual search. J Neurophysiol 80:2918-2940.

Deco G, Zihl J (2001) A neurodynamical model of visual attention: feedback enhancement of spatial resolution in a hierarchical system. J Comput Neurosci 10:231-253.

Eimer M (1996) The N2pc component as an indicator of attentional selectivity. Electroencephalogr Clin Neurophysiol 99:225-234.

Fink GR, Halligan PW, Marshall JC, Frith CD, Frackowiak RS, Dolan RJ (1996) Where in the brain does visual attention select the forest and the trees? Nature 382:626-628.

Fuchs M, Wagner M, Köhler T, Wischmann HA (1999) Linear and nonlinear current density reconstructions. J Clin Neurophysiol 16:267-295.

Hämäläinen M, Ilmoniemi RJ (1984) Interpreting measured magnetic fields of the brain: estimates of current distributions. Technical Report TKK-F-A559. Helsinki, Finland: Helsinki University of Technology.

Hämäläinen MS, Sarvas J (1989) Realistic conductivity geometry model of the human head for interpretation of neuromagnetic data. IEEE Trans Biomed Eng 36:165-171.

Han S, Weaver JA, Murray SO, Kang X, Yund EW, Woods DL (2002) Hemispheric asymmetry in global/local processing: effects of stimulus position and spatial frequency. Neuroimage 17:1290-1299.

Heinze HJ, Münte TF (1993) Electrophysiological correlates of hierarchical stimulus processing: dissociation between onset and later stages of global and local target processing. Neuropsychologia 31:841-852.

Hickey C, Di Lollo V, McDonald JJ (2009) Electrophysiological indices of target and distractor processing in visual search. J Cogn Neurosci 21:760-775.

Hillyard SA, Vogel EK, Luck SJ (1998) Sensory gain control (amplifica- tion) as a mechanism of selective attention: electrophysiological and neuroimaging evidence. Philos Trans R Soc Lond B Biol Sci 353:1257-1270.

Hochstein S, Ahissar M (2002) View from the top: hierarchies and reverse hierarchies in the visual system. Neuron 36:791-804.

Hopf J-M, Luck SJ, Girelli M, Hagner T, Mangun GR, Scheich H, Heinze H-J (2000) Neural sources of focused attention in visual search. Cereb Cortex 10:1233-1241.

Hopf J-M, Boelmans K, Schoenfeld AM, Heinze H-J, Luck SJ (2002) How does attention attenuate target-distractor interference in vision? Evidence from magnetoencephalographic recordings. Brain Res Cogn Brain Res 15:17-29.

Hopf J-M, Boehler CN, Luck SJ, Tsotsos JK, Heinze H-J, Schoenfeld MA (2006a) Direct neurophysiological evidence for spatial suppression surrounding the focus of attention in vision. Proc Natl Acad Sci U S A 103:1053-1058.

Hopf J-M, Luck SJ, Boelmans K, Schoenfeld MA, Boehler CN, Rieger J, Heinze H-J (2006b) The neural site of attention matches the spatial scale of perception. J Neurosci 26:3532-3540.

Hopf J-M, Boehler CN, Schoenfeld MA, Heinze H-J, Tsotsos JK (2010) The spatial profile of the focus of attention in visual search: insights from MEG recordings. Vision Res 50:1312-1320.

Kastner S, De Weerd P, Pinsk MA, Elizondo MI, Desimone R, Ungerleider LG (2001) Modulation of sensory suppression: implications for receptive field sizes in the human visual cortex. J Neurophysiol 86:1398-1411.

Lamme VAF, Roelfsema PR (2000) The distinct modes of vision offered by feedforward and recurrent processing. Trends Neurosci 23:571-579.

Leblanc E, Prime DJ, Jolicoeur P (2008) Tracking the location of visuospatial attention in a contingent capture paradigm. J Cogn Neurosci 20:657-671.

Luck SJ (2005) An introduction to the event-related potential technique. Cambridge, MA: MIT Press.

Luck SJ, Hillyard SA (1994) Spatial filtering during visual search: evidence from human electrophysiology. J Exp Psychol Hum Percept Perform 20:1000-1014.

Luck SJ, Hillyard SA (1995) The role of attention in feature detection and conjunction discrimination: an electrophysiological analysis. Int J Neurosci 80:281-297.

Luck SJ, Fan S, Hillyard S (1993) Attention-related modulation of sensory-evoked brain activity in a visual search task. J Cogn Neurosci 5:188-195.

Luck SJ, Girelli M, McDermott MT, Ford MA (1997) Bridging the gap between monkey neurophysiology and human perception: an ambiguity resolution theory of visual selective attention. Cogn Psychol 33:64-87.

Martínez A, DiRusso F, Anllo-Vento L, Sereno MI, Buxton RB, Hillyard SA (2001) Putting spatial attention on the map: timing and localization of stimulus selection processes in striate and extrastriate visual areas. Vision Res 41:1437-1457.

Maunsell JH, Cook EP (2002) The role of attention in visual processing. Philos Trans R Soc Lond B Biol Sci 357:1063-1072.

Mehta AD, Ulbert I, Schroeder CE (2000) Intermodal selective attention in monkeys. I: distribution and timing of effects across visual areas. Cereb Cortex 10:343-358.

Müller NG, Kleinschmidt A (2004) The attentional 'spotlight's' penumbra: center-surround modulation in striate cortex. Neuroreport 15:977-980.

Noesselt T, Hillyard SA, Woldorff MG, Schoenfeld A, Hagner T, Jäncke L, Tempelmann C, Hinrichs H, Heinze H-J (2002) Delayed striate cortical activation during spatial attention. Neuron 35:575-587.

Pinsk MA, Doniger GM, Kastner S (2004) Push-pull mechanism of selective attention in human extrastriate cortex. J Neurophysiol 92:622-629.

Rees G, Frith CD, Lavie N (1997) Modulating irrelevant motion perception by varying attentional load in an unrelated task. Science 278:1616-1619.

Robinson SE (1989) Environmental noise cancellation for biomagnetic measurements. In: Advances in biomagnetism (Williamson SJ, Hoke M, Stroink G, Kotani M, eds), pp 721-724. New York: Plenum.

Schwartz S, Vuilleumier P, Hutton C, Maravita A, Dolan RJ, Driver J (2005) 
Attentional load and sensory competition in human vision: modulation of fMRI responses by load at fixation during task-irrelevant stimulation in the peripheral visual field. Cereb Cortex 15:770-786.

Serences JT, Yantis S, Culberson A, Awh E (2004) Preparatory activity in visual cortex indexes distractor suppression during covert spatial orienting. J Neurophysiol 92:3538-3545.

Slotnick SD, Hopfinger JB, Klein SA, Sutter EE (2002) Darkness beyond the light: attentional inhibition surrounding the classic spotlight. Neuroreport 13:773-778.

Smith AT, Singh KD, Greenlee MW (2000) Attentional suppression of activity in the human visual cortex. Neuroreport 11:271-277.

Spratling MW, Johnson MH (2004) A feedback model of visual attention. J Cogn Neurosci 16:219-237.
Tsotsos JK, Culhane SM, Wai WYK, Lai Y, Davis N, Nuflo F (1995) Modeling visual attention via selective tuning. Artif Intell 78:507-545.

Tsotsos JK, Rodríguez-Sánchez AJ, Rothenstein AL, Simine E (2008) The different stages of visual recognition need different attentional binding strategies. Brain Res 1225:119-132.

Vanduffel W, Tootell RBH, Orban GA (2000) Attention-dependent suppression of metabolic activity in early stages of the macaque visual system. Cereb Cortex 10:109-126.

Vogel EK, Luck SJ, Shapiro KL (1998) Electrophysiological evidence for a postperceptual locus of suppression during the attentional blink. J Exp Psychol Hum Percept Perform 24:1656-1674.

Woodman GF, Luck SJ (1999) Electrophysiological measurement of rapid shifts of attention during visual search. Nature 400:867-869. 